Article

\title{
Computational Study of Quasi-2D Liquid State in Free Standing Platinum, Silver, Gold, and Copper Monolayers ${ }^{+}$
}

\author{
Li-Ming Yang ${ }^{1, *}$, Ariel B. Ganz ${ }^{2}$, Matthew Dornfeld ${ }^{3}$ and Eric Ganz ${ }^{3, *}$ \\ 1 School of Chemistry and Chemical Engineering, Huazhong University of Science and Technology, \\ Wuhan 430074, China \\ 2 Division of Nutritional Sciences, Cornell University, 339 Savage Hall, Ithaca, NY 14853, USA \\ 3 Department of Physics, University of Minnesota, 115 Union St., SE, Minneapolis, MN 55416, USA \\ * Correspondence: lmyang.uio@gmail.com (L.-M. Y.); ganzx001@umn.edu (E.G.) \\ $\dagger$ Dedicated to Prof. Thomas Frauenheim on the occasion of his 65th birthday.
}

Academic Editor: Annette Bussmann-Holder

Received: 3 November 2015; Accepted: 13 February 2016; Published: 2 March 2016

\begin{abstract}
Recently, freestanding atomically thick Fe metal patches up to 10 atoms wide have been fabricated experimentally in tiny pores in graphene. This concept can be extended conceptually to extended freestanding monolayers. We have therefore performed $a b$ initio molecular dynamics simulations to evaluate the early melting stages of platinum, silver, gold, and copper freestanding metal monolayers. Our calculations show that all four freestanding monolayers will form quasi-2D liquid layers with significant out-of-plane motion and diffusion in the plane. Remarkably, we observe a $4 \%$ reduction in the Pt most likely bond length as the system enters the liquid state at $2400 \mathrm{~K}$ (and a lower effective spring constant), compared to the system at 1200 and $1800 \mathrm{~K}$. We attribute this to the reduced average number of bonds per atom in the Pt liquid state. We used the highly accurate and reliable Density Functional Theory (DFT-D) method that includes dispersion corrections. These liquid states are found at temperatures of $2400 \mathrm{~K}, 1050 \mathrm{~K}, 1600 \mathrm{~K}$, and $1400 \mathrm{~K}$ for platinum, silver, gold, and copper respectively. The pair correlation function drops in the liquid state, while the bond orientation order parameter is reduced to a lesser degree. Movies of the simulations can be viewed online (see Supplementary Material).
\end{abstract}

Keywords: 2-D melting; molecular dynamics; density functional theory; 2-D liquid

\section{Introduction}

Historically, it has been difficult to fabricate freestanding monolayer metal films experimentally. However, recently, freestanding atomically thick iron membranes suspended in and supported by tiny graphene pores have been fabricated [1]. These iron layers were only up to 10 atoms wide. This is an exciting development, but due to the presence of the pores around the periphery, the iron patches differed structurally from the predictions for an extended freestanding 2D monolayer. This finding demonstrates the potential of perforated graphene as a support for small 2D monolayers, and paves the way for novel freestanding 2D structures to be formed. Zhao et al. used density functional theory (DFT) calculations to study these systems, and predicted that the largest thermodynamically stable patch would be 12 atoms across [1]. In our paper, we will be studying freestanding systems of quasi-2D metal atoms with significant out-of-plane motions, using highly accurate Density Functional Theory with dispersion corrections (DFT-D).

The use of small pores in graphene to support metals could potentially be used to fabricate different metal patches or small freestanding metal monolayers. Following the discovery of iron 
patches in graphene pores, this method has been extended computationally to small gold patches in the pores [2]. Koskinen and Korhonen $(\mathrm{K} \& \mathrm{~K})$ studied the solid and liquid phases of a small monolayer gold patch in a graphene hole using primarily the less reliable density-functional tight-binding (DFTB) method [2]. This 49-atom gold patch stayed solid up to $700 \mathrm{~K}$, and then at $900 \mathrm{~K}$ formed an unusual quasi-2D liquid layer. In fact, $\mathrm{K} \& \mathrm{~K}$ also extended this study to a close packed quasi-2D monolayer of gold in a periodic cell. They also checked this result using more reliable DFT. Remarkably, they observed quasi-2D liquid behavior, and predicted a melting point of $1200 \mathrm{~K}$.

In recent years, there has been tremendous interest in two-dimensional materials due to their intriguing properties and potential applications in nanoscience and nanotechnology [3]. 2D materials have a much larger surface area compared to the bulk phase, leading to unique thermal, electrical, and mechanical properties. Melting of 2D materials is substantially different than melting in the bulk. For example, in three dimensions, theoretically one can define the melting point as the boundary between the Gibbs free energy minima of the solid and liquid phases. For two dimensions, various criteria have been proposed. A modified Lindemann criterion for use in 2D was proposed by Zheng et al. [4].

More recently, a dynamic criteria for melting in two dimensions was proposed by Zahn and Maret [5]. Freestanding 2D sheets exhibit significant motion out of the plane in addition to the introduction of defects and long range corrugations as the temperature is raised. In this paper, we will refer to systems with significant out-of-plane motion as quasi-2D. Zakharchenko et al. have studied the high temperature behavior of graphene using atomistic simulations [6], and Singh et al. have studied the vibrational stability and melting behavior of freestanding $\mathrm{MoS}_{2}$ [7]. Los et al. predict a more accurate melting point of $\mathrm{T}_{\mathrm{m}}=4510 \mathrm{~K}$ for graphene using nucleation theory, which is about $250 \mathrm{~K}$ higher than that of graphite, and so far the highest of all materials [8]. A melting point of $3700 \mathrm{~K}$ has been predicted for single layer freestanding MoS $_{2}$ [7]. In 2015, Merino and coworkers predicted the melting of boron 40 molecules in DFT simulations. They consider this system to be a nanobubble, and observed diffusion of individual atoms in the shell [9].

There is a long history of the study of melting in two dimensions. In the 1970s, the Kosterlitz-Thouless theory was developed [10]. According to the Kosterlitz-Thouless-HalperinNelson-Young (KTHNY) theory, the melting mechanism of 2D crystals proceeds via two consecutive continuous transitions, caused by the creation of topological defects [10-12]. One characteristic of the melting of many 2D systems is the existence of a distinct hexatic phase. This phase shows quasi-long-range bond orientational order, even as the positional order becomes short range. A recent study of 2D hard disks found unusual two-stage melting [13]. A follow up study on quasi-2D hard spheres with significant out-of-plane particle motions [14] found that the peculiar two-stage melting scenario of a continuous solid-hexatic and a first-order hexatic-liquid transition, as observed for a truly 2D system of hard disks, persists [13]. Dijkstra and coworkers found that the hexatic phase was only observed in a narrow range of parameters [14].

The individual bond strength of these freestanding $2 \mathrm{D}$ metal layers at $0 \mathrm{~K}$ is larger than in the bulk due to the reduction in total bonds per atom (6 versus 12). For Ag, the individual bond strength actually increases from $0.42 \mathrm{eV}$ for the bulk to $0.66 \mathrm{eV}$ for the freestanding 2D layer [15]. The 2D Ag monolayer and the 3D bulk have cohesive energies of 2.01 and $2.49 \mathrm{eV}$ atom, respectively [15]. We have also studied the properties of the freestanding Au monolayer [16]. We observe an increase in bond strength from $0.52 \mathrm{eV} /$ bond for the 3D bulk gold to 0.94 for the freestanding 2D monolayer [16]. The increased bond strength of 2D layers also explains why the melting point of graphene is higher than that of diamond [8].

The melting points of the bulk materials correlate with the bond strengths of these materials. For example, the bulk $\mathrm{Cu}, \mathrm{Ag}$, $\mathrm{Au}$, and Pt cohesive energies are 3.48, 2.49, 3.11, and $5.84 \mathrm{eV}$, respectively [17]. We see that platinum has the highest cohesive energy. The bulk melting points are 1356, 1235, 1338, and $2041 \mathrm{~K}$, respectively [18]. We expect that the temperatures of the liquid states or melting points of the freestanding quasi-2D monolayers will also scale with cohesive energy and bond strength. We see that platinum also has the highest bulk melting point. Therefore, we can expect that the freestanding 
2D platinum monolayer should be the most stable of the four materials. Note that bulk platinum is also a noble metal, and therefore the 2D platinum monolayer may be relatively durable and resistant and thus more favorable for experimental fabrication.

In this paper, we use ab initio molecular dynamics simulations to study freestanding quasi-2D monolayers of platinum, silver, gold, and copper. We will use freestanding periodic $8 \times 8$ simulations, the results of which can be applied to a broad range of situations. Furthermore, the isolated freestanding monolayer is of fundamental interest. Real experimental materials will, of course, need to be supported in some way. For example, either a monolayer could be grown on a substrate, which is then etched away underneath to leave a freestanding section, or a freestanding monolayer could be transferred onto a substrate over a small hole. Alternatively, metal atoms could be added to a graphene layer with tiny holes to form embedded patches as has been demonstrated by Zhao et al. [1]. We believe that our results should have general application to a variety of situations, and contribute beyond the existing studies of tiny patches embedded holes in graphene.

We use the DFT-D method, which includes dispersion corrections that are necessary for the long bond distances found in these systems. We calculated the diffusion and particle correlation functions to demonstrate that the layers are liquid at specific temperatures. Analysis of bond numbers and bond lengths revealed unexpected results with the platinum layer showing unusually low bond lengths in the liquid state. The pair correlation function drops in the liquid state, while orientational order is reduced to a lesser degree. Movies of the simulations can be viewed online.

\section{Computational Methods}

$A b$ initio Born-Oppenheimer molecular dynamics simulations were performed. A periodic boundary condition unit cell that holds an $8 \times 8$ array of atoms $(21.0 \times 21.0 \times 15.0 \AA$ for $\mathrm{Pt})$ was used. One unit cell in the $X-Y$ plane along with the outline of the periodic cell are shown in Figure 1. The 15- $\AA$ spacing in $\mathrm{Z}$ between layers ensures good isolation in each layer. For $\mathrm{Cu}$, $\mathrm{Ag}$, and $\mathrm{Au}$, the edge dimensions of the unit cells were $19.3 \AA, 22.3 \AA$, and $21.86 \AA$, respectively. The scalar-relativistic DFT-D and Tkatchenko-Scheffler (TS) method were used in CASTEP [19] in Materials Studio 7 and 8. Molecular Dynamics (MD) simulation in the constant number, volume, temperature (NVT) ensemble were carried out for 6-27 ps with a time step of $2.5 \mathrm{fs}$ for gold and copper, and $4 \mathrm{fs}$ for silver and platinum. The parameters were: Accuracy $=$ fine; Self-Consistent Field $(\mathrm{SCF})=3 \times 10^{-6}$; Smearing $=0.04$; Direct Inversion of the Iterative Subspace method (DIIS) $=20$; Nosé-Hoover method [20], Nosé $Q=2$, Nosé chain length $=2$; Fixed center of mass. For the higher temperatures, the first 12 ps of each run were considered an equilibration period (less time was needed for the lower temperatures). More details on each run are in the Supplementary Information (SI). Data were acquired after the equilibration. Materials Studio was also used to create the initial structures and visualize the results. The Root Mean Square Displacement (RMSD) was calculated using the differences between an initial data frame (after equilibration), and the final frame of each simulation.

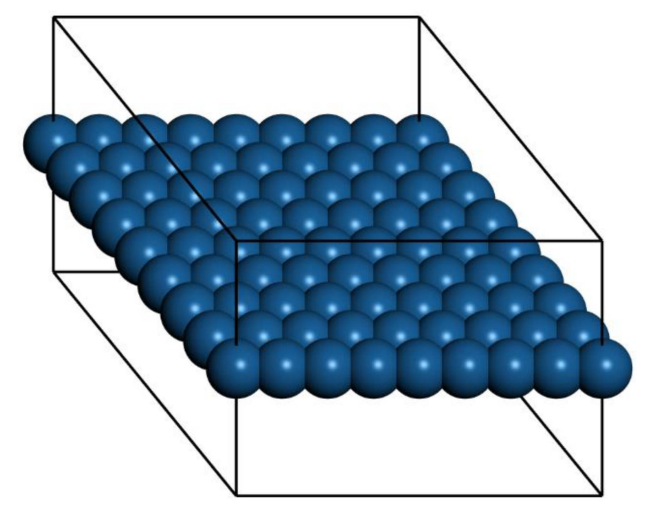

Figure 1. Single unit cell of freestanding $8 \times 8 \mathrm{Pt}$ model at $0 \mathrm{~K}$. 
The pair correlation function for our quasi-2D system $g(r)$ was calculated, where $\sigma$ is the two-dimensional atomic density, $\mathrm{N}$ is the total number of atoms, and $\delta$ is the delta function.

$$
g(r)=\frac{1}{2 \pi r \sigma N}\left\langle\sum_{i} \sum_{j \neq i} \delta\left(r-r_{i j}\right)\right\rangle
$$

The large vacuum space between layers assures good isolation of the freestanding quasi-2D layer. The diffusion coefficient $\mathrm{D}$ was calculated using $\left\langle r^{2}\right\rangle=4 \mathrm{Dt}$. The $\mathrm{R}$ computing environment [21] and Python programming environment [22] were used for plot generation and data analysis. The RMSD plots at higher temperatures were fit to $\mathrm{y}=\sqrt{a+b t}$, to include both $\mathrm{Z}$ motion and $\mathrm{X}-\mathrm{Y}$ diffusion. The Forcite analysis program was used to generate velocity correlation functions in Materials Studio. Origin 2015 was also used to make some plots. The bond orientation correlation function was calculated by locating the nearest neighbors for each atom in each frame (atoms within 1.38 times the distance $\mathrm{P}=2.76,3.06,2.88$, and $2.56 \AA$, respectively, for copper, silver, gold, and platinum). The $2 \mathrm{D}$ bond angle was then determined by ignoring the $\mathrm{Z}$ coordinate of each atom, and then determining the angle compared to the $X$-axis. Each bond centroid was calculated. Then, a modified distribution function $g 6(r)=\langle\cos (6 \theta)\rangle$, where $\theta$ is the difference between two bond angles, and $r$ is the full 3D distance between two bond centroids was calculated. The factor of six ensures that six-fold symmetry of the perfect close packed layer will produce a result of one.

The bond length distributions have been fit to a Boltzmann distribution shown in Equation (2). We approximate the energy $E$ of the bond length deviation $\left(\ell-\ell_{0}\right)$ using a simple spring model with spring constant $k_{\text {spring }}$ at low deviations, as shown in Equation (3). Here, $\ell$ is the bond length, and $\ell_{0}$ is the most probable bond length at a given temperature, and corresponds to zero spring extension. We have also included a hard sphere repulsive term $\left[\ell-r_{0}\right]^{12}$ with $C$ and $r_{0}$ determined empirically for each plot.

$$
\begin{gathered}
P=A e^{-E / k_{B} T} \\
E=\frac{1}{2} k_{\text {spring }}\left[\ell-\ell_{0}\right]^{2}+\mathrm{C} /\left[\ell-r_{0}\right]^{12} .
\end{gathered}
$$

\section{Results and Discussion}

We performed a set of $a b$ initio Born-Oppenheimer molecular dynamics simulations to study the melting properties of freestanding quasi-2D Pt, $\mathrm{Ag}, \mathrm{Au}$, and $\mathrm{Cu}$ monolayers. The results are organized as follows. For all of the systems at low temperatures, the system maintains the framework during the simulations. Long wavelength oscillations are observed in side views, and then larger amplitudes and shorter wavelength oscillations are observed as the temperatures are raised (snapshots showing side views are included in the SI).

At intermediate temperatures, larger oscillations in the $\mathrm{Z}$ direction were observed as well as substantial bond length extensions; however, the atoms oscillate back and forth without any net diffusive motion or atomic switching. The hexagonal close pack (HCP) arrangement is maintained and each atom has six bonds. In this temperature range, the bond length extensions are very large, ranging from $20 \%-36 \%$. We note that such large bond length extensions could be associated with bulk melting at real world time scales and in experimental conditions. However, the freestanding quasi-2D metal films we are discussing in this paper are a very different case, and must be studied independently.

Finally, at higher temperatures, we observe the beginnings of diffusive motion, and atom switching. It is in this temperature range that we observe quasi-2D melting behavior (we will call this the quasi-2D liquid regime). In some cases, we see individual adatoms on top of the layer. Each material is discussed below in detail, with a focus on the quasi-2D melting and quasi-2D liquid behavior below. If we raise the temperature beyond this regime, the monolayers become unstable, and we see larger 3D structures forming leaving behind large holes. 


\subsection{Platinum}

The range from $2300 \mathrm{~K}$ to $2400 \mathrm{~K}$ represents the quasi-2D liquid regime for platinum. At $2300 \mathrm{~K}$, we see an increasing number of small holes, and the atoms are now able to diffuse. At $2400 \mathrm{~K}$, we see increasing amounts of diffusion, and the system has larger holes, and more excursions in the $Z$ direction. Snapshots from the simulation are shown in Figure 2. A ball and stick model is also shown in the SI to highlight the binding configuration and holes. A movie of the $2400 \mathrm{~K} \mathrm{Pt}$ simulation is shown in the SI, and one can see that the atoms are moving vigorously.

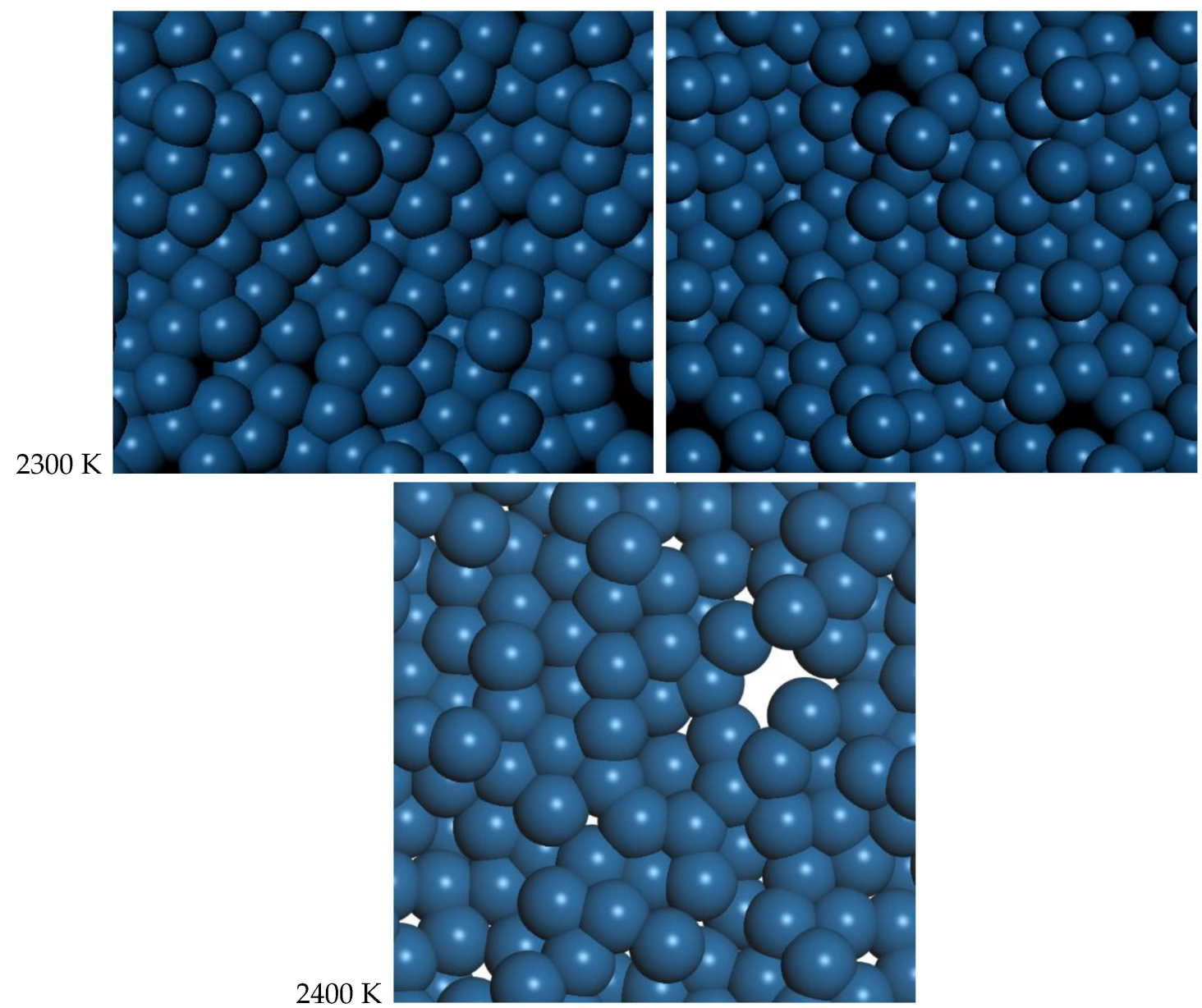

Figure 2. Molecular dynamics snapshots of quasi-2D Pt layer. We show two snapshots at $2300 \mathrm{~K}$, separated by $0.2 \mathrm{ps}$ and one snapshot at $2400 \mathrm{~K}$. Atoms are mobile and holes come and go. This is quasi-2D liquid behavior.

We see that the pair correlation function for the quasi-2D Pt layer drops away quickly at 2300 and $2400 \mathrm{~K}$ (see Figure 3). We see that the two next nearest neighbor peaks near $5 \AA$ merge together at $2300 \mathrm{~K}$ and above. For lower temperatures, at and below $2200 \mathrm{~K}$, the framework is maintained, although there is substantial motion in the $\mathrm{Z}$ direction and substantial bond length extension.

In Figure 4, we show the bond length distribution for freestanding quasi-2D Pt monolayer at $1200 \mathrm{~K}, 1800 \mathrm{~K}$, and $2400 \mathrm{~K}$. At $1200 \mathrm{~K}$ and $1800 \mathrm{~K}$, the framework is maintained, but extensive motion is observed in the $\mathrm{Z}$ direction. The bond length distribution is almost all well above the $0 \mathrm{~K}$ bond length of $2.63 \AA$ (shown as the short vertical line below the curves). However, at $2400 \mathrm{~K}$ in the liquid state, due to the presence of holes, the average number of bonds per atom is reduced from 6 to 5.1. This can also be seen clearly in the ball and stick model at this temperature shown in the SI. 


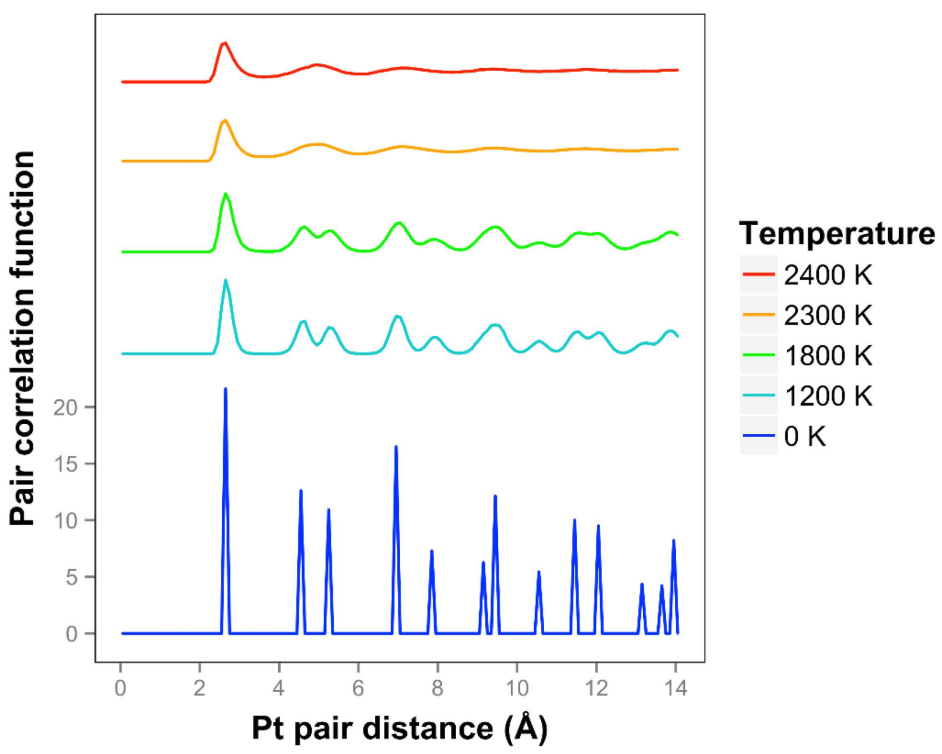

Figure 3. Pair correlation function for freestanding platinum quasi-2D monolayer at temperatures ranging from $0 \mathrm{~K}$ to $2400 \mathrm{~K}$. At $2300 \mathrm{~K}$ and $2400 \mathrm{~K}$ the pair correlation function drops off quickly, and the freestanding quasi-2D Pt monolayer is a quasi-2D liquid. Higher temperatures are offset for visibility.

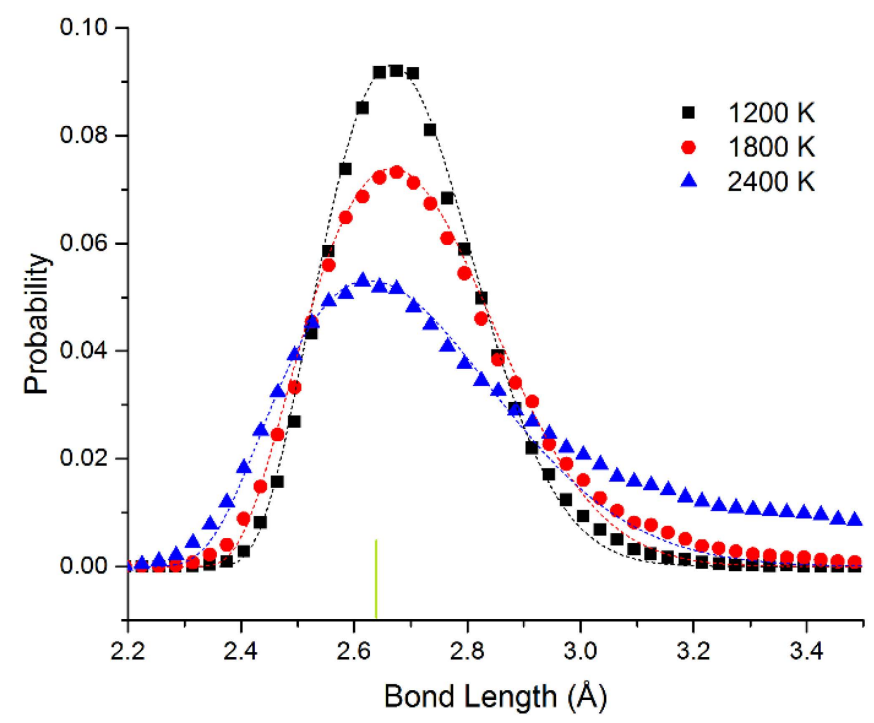

Figure 4. Bond length distribution for freestanding quasi-2D Pt monolayer at $1200 \mathrm{~K}, 1800 \mathrm{~K}$, and $2400 \mathrm{~K}$. The $0 \mathrm{~K}$ bond length of $2.63 \AA$ is shown as a short vertical green line below the curves. At $1200 \mathrm{~K}$ and $1800 \mathrm{~K}$, almost all of the bond lengths are well above the $0 \mathrm{~K}$ value. The most likely length shrinks by $4 \%$ at $2400 \mathrm{~K}$ in the liquid state, compared to 1200 and $1800 \mathrm{~K}$. Solid lines are fitted to the Boltzmann distribution as discussed in the text.

In Figure 5a, we show the results of the RMSD calculations for the platinum simulations. We see that at the lower temperatures of $1200 \mathrm{~K}$ and $1800 \mathrm{~K}$, there is no net diffusion, and the RMSD has a relatively constant value of approximately $1 \AA$ Aver times of 8-12 ps. In this case, we expect the RMSD to be mainly due to the motion of the atoms moving in the $\mathrm{Z}$ direction, with amplitude dependent on temperature, although there is no net diffusion in the plane yet during the simulation times. At higher temperatures $(2300 \mathrm{~K}$ and $2400 \mathrm{~K})$, where the atoms are starting to diffuse and are more free to move to different locations (in the quasi-2D liquid regime), we see that the RMSD is increasing with time. 
In these cases, at longer times we observe the expected 2D random walk diffusion characteristic. At the higher temperatures of 2300 and $2400 \mathrm{~K}$, where we are in the quasi-2D liquid regime, we observe net diffusion consistent with random walk behavior. We find RMSD values of $5.7 \AA$ and $3.2 \AA$ after 20 and 5 ps, respectively. We can estimate the diffusion coefficient $\mathrm{D}=0.4 \AA^{2} / \mathrm{ps}$ for $2300 \mathrm{~K}$, and $0.5 \AA^{2} /$ ps for $2400 \mathrm{~K}$. The velocity auto correlation function for $\mathrm{Pt}$ at $2400 \mathrm{~K}$ is shown in the SI. We observe that the correlation dissipates quickly after less than 1 ps. In Figure 5b, we also show the bond orientation order parameter for platinum. We see that this is close to one for short distances and lower temperatures, and is reduced as we raise the temperature. Although the value drops somewhat with distance, we see that bond orientational order is maintained over the longest distance available in the simulation. For $2400 \mathrm{~K}$, where we are in the liquid state, we see an average value of around 0.63-0.67.
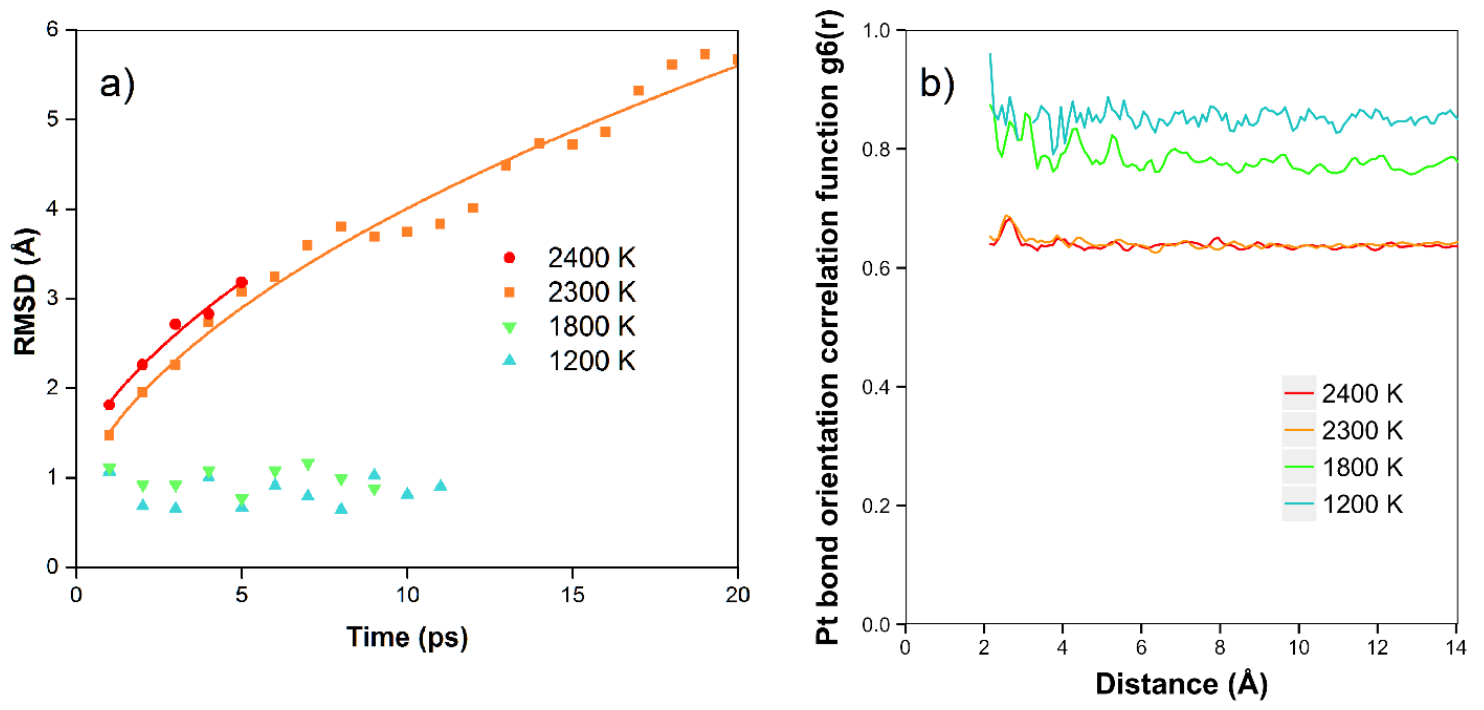

Figure 5. (a) Root mean square displacement (RMSD, in $\AA$ ) for platinum simulations at $1200-2400 \mathrm{~K}$. At lower temperatures, the RMSD is constant around $1 \AA$ due to oscillation primarily in the $\mathrm{Z}$ direction. At 2300 and $2400 \mathrm{~K}$, we see extended diffusion. (b) Bond orientation correlation function.

The data points have been fitted to the Boltzmann distribution shown in Equation (2). We approximate the energy $E$ of the bond length deviation $\left(\mathrm{d}-\mathrm{d}_{0}\right)$ using a simple spring model with spring constant $\mathrm{k}_{\text {spring }}$ at low deviations as shown in Equation (3) (with an additional hard sphere repulsive term). For Pt, we see that the effective spring constant $\mathrm{k}_{\text {spring }}=4.3$ and $4.1 \mathrm{eV} / \AA^{2}$ at 1200 and $1800 \mathrm{~K}$. The fits are quite good at all temperatures. At $2400 \mathrm{~K}$, the system changes to the liquid state. This state has $4 \%$ shorter bond length at the maximum. We attribute the shortened bond lengths to the lower number of bonds in the liquid state. We observe that the effective spring constant decreases to $\mathrm{k}_{\text {spring }}=2.8 \mathrm{eV} / \AA^{2}$. This shrinking as we raise the temperature corresponds to a negative coefficient of thermal expansion as we enter the liquid state. At $2400 \mathrm{~K}$, at long bond lengths, there are significant excess observations from 2.8 to $3.5 \AA$. This is expected as we approach the van der Waals limit as the atom moves away from the plane of the other atoms (the bonding becomes weaker than the spring model and more atoms will be observed with these long bond lengths).

We also explored the effect of raising the simulation size to $10 \times 10$ atoms for the platinum model. A single run at $2150 \mathrm{~K}$ for 21 ps gave similar results to the $8 \times 8$ models at $2300-2400 \mathrm{~K}$.

\subsection{Gold}

For gold, the quasi-2D liquid regime is $1400-1600 \mathrm{~K}$. For this range of temperatures, we see the quasi-2D layer is maintained, but also occasional small holes and adatoms are observed. Snapshots from the molecular dynamics simulations at $1400 \mathrm{~K}$ and $1600 \mathrm{~K}$ are shown in Figure 6 . A movie of the $1600 \mathrm{~K} \mathrm{Au}$ simulation is shown in the SI. At $1400 \mathrm{~K}$ and $1600 \mathrm{~K}$, diffusion is observed, with typical 
RMSD = 2.4 $\AA$ and $3.4 \AA$ after 12 ps, respectively (see Figure S7). These displacements give diffusion coefficients of $\mathrm{D}=0.12$ and $0.25 \AA^{2} / \mathrm{ps}$, respectively. These can be compared to the result of $0.14 \AA^{2} / \mathrm{ps}$ at $1600 \mathrm{~K}$ from K \& K [2]. At $1600 \mathrm{~K}$, the average number of bonds is 5.5, somewhat reduced compared to the low temperature results, but still larger than the Pt results at $2400 \mathrm{~K}$.

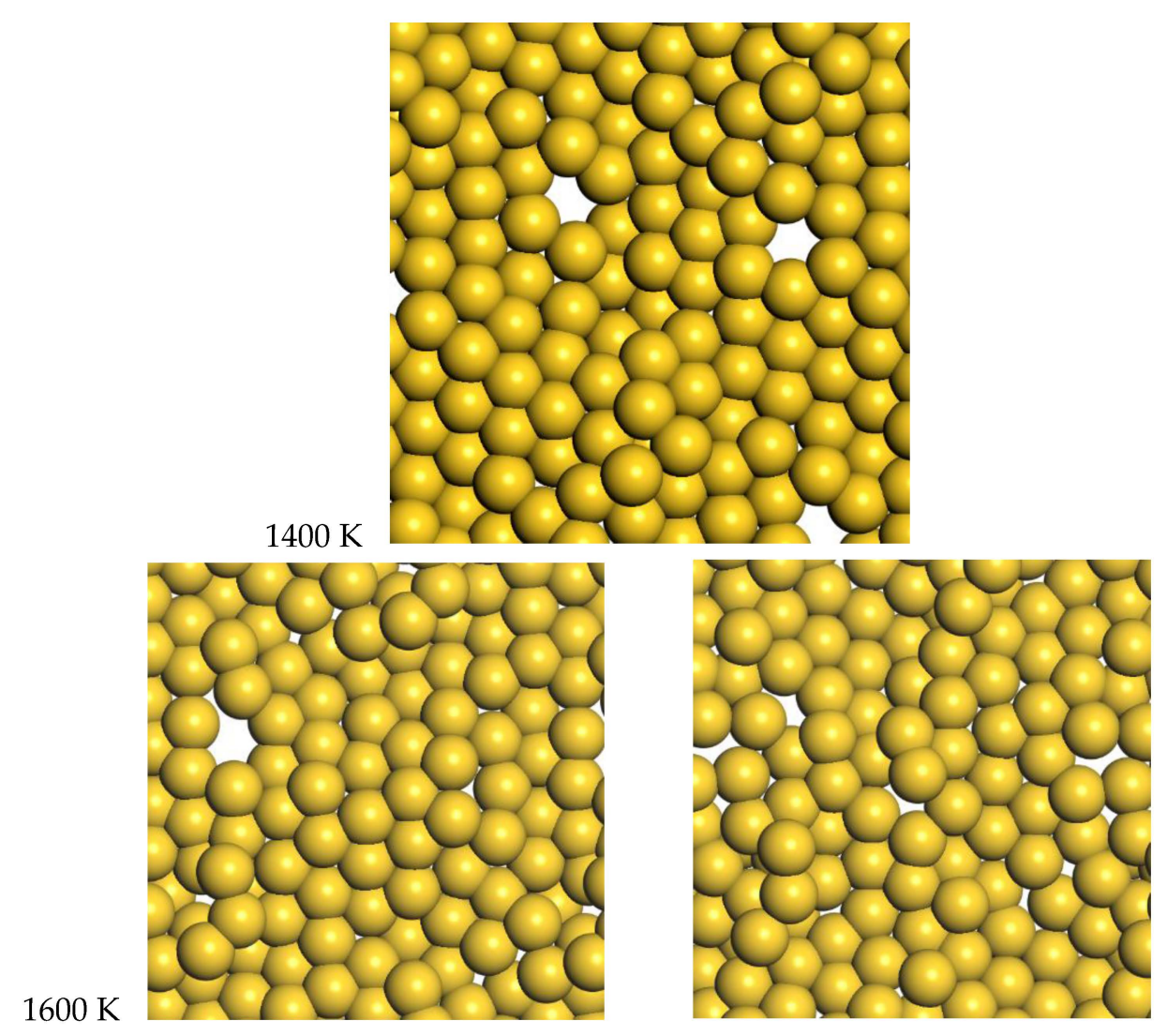

Figure 6. Molecular dynamics snapshots of quasi-2D Au layers at $1400 \mathrm{~K}$ and $1600 \mathrm{~K}$. At $1600 \mathrm{~K}$, we show two frames separated by 0.5 ps. quasi-2D liquid behavior is observed at these temperatures.

We also raised the temperature to $2000 \mathrm{~K}$, but at this temperature the gold layer is unstable and falls apart. Large holes are left behind and nanowires or nanobridges form. A snapshot from the end of the simulation is shown in the Supplementary Information (SI).

Looking at the pair correlation function for the quasi-2D gold layers (shown in Figure 7), we observe that the correlation is significantly reduced as the temperature is increased. At longer distances, the correlation flattens out and drops away. The two next nearest neighbor peaks near $5 \AA$ merge together at temperatures of $1400 \mathrm{~K}$ and above. We can compare our correlation results to the previous results of $K \& \mathrm{~K}$ [2]. The shapes of the curves are quite different, and it should be noted that they are using the less accurate DFTB theory. They identified a melting point of $1200 \mathrm{~K}$. Considering the disappearance of the two next nearest neighbor lines and the general disappearance at longer distances, a comparable temperature for our results would be $1400 \mathrm{~K}$. By including the diffusive interactions (which are very important for our system with such long bond length extensions and in the liquid regime), and using a more accurate DFT-D theory, we see a substantial $20 \%$ increase in temperature compared to the $\mathrm{K} \& \mathrm{~K}$ results. Note that $\mathrm{K} \& \mathrm{~K}$ also did DFT calculations on these Au models and included movies but not correlation functions of the results. 


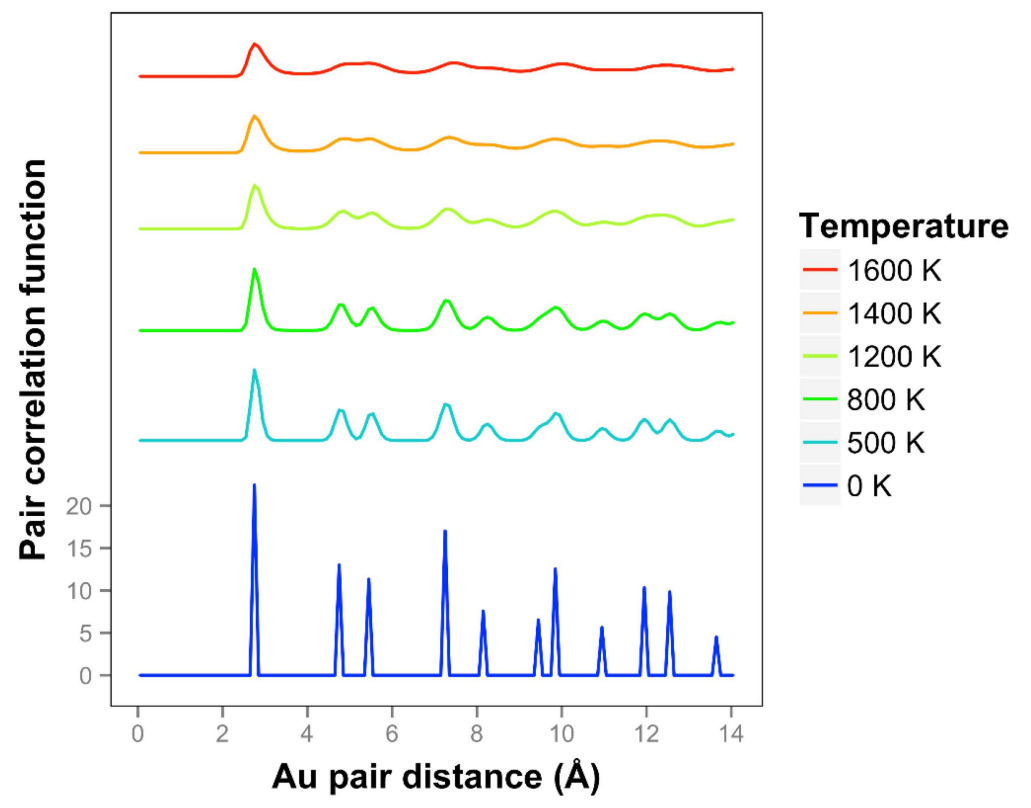

Figure 7. Pair correlation function for gold quasi-2D layer at temperatures ranging from $0 \mathrm{~K}$ to $1600 \mathrm{~K}$.

Higher temperatures are offset for visibility.

The cell dimensions were set using $0 \mathrm{~K}$ optimization. We tested the thermal expansion from $0 \mathrm{~K}$ up to the liquid state temperatures (this question was also explored by $\mathrm{K} \& \mathrm{~K}$ for gold). We find a roughly $3 \% 2 \mathrm{D}$ areal expansion leading to a $1.7 \%$ linear change in dimensions. We did run some simulations with a $1.7 \%$ linearly expanded cell, but the results were unchanged. The large $\mathrm{Z}$ motions dominated these simulations; thus, a small $1.7 \%$ linear change in cell dimension is not significant. An insignificant amount of stress is imposed as the atoms are moving back and forth in the $\mathrm{Z}$ direction.

\subsection{Silver}

For Ag, the quasi-2D liquid regime is near $1050 \mathrm{~K}$. Snapshots from this temperature are shown in Figure 8, and a movie is shown in the SI. At $1200 \mathrm{~K}$, we observe the conversion of the quasi-2D layer to lower energy nanorod or 3D bridge structures with 3D bonding (this is the unstable regime). This behavior is similar to the formation of $1 \mathrm{D}$ gold nanowires by thinning in a Transmission Electron Microscope [23].

$1050 \mathrm{~K}$
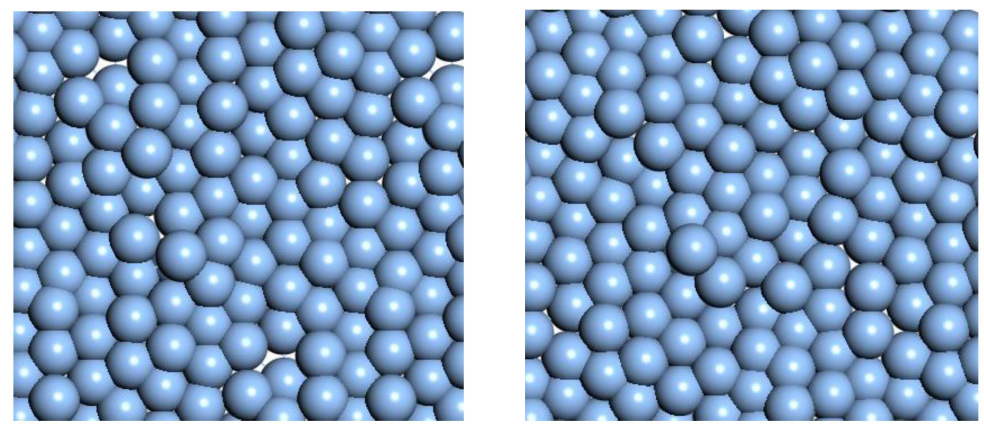

Figure 8. Cont. 


\section{$1200 \mathrm{~K}$}

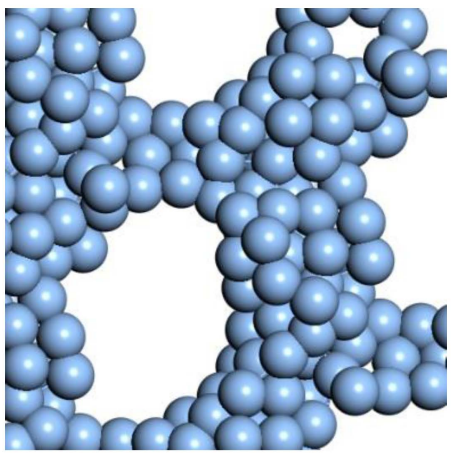

Figure 8. Snapshots of quasi-2D Ag layer at $1050 \mathrm{~K}$ and $1200 \mathrm{~K}$.

At $1050 \mathrm{~K}$ (where we observe quasi-2D liquid behavior in our timeframe), we see that the pair correlation function (Figure 9) is substantially reduced compared to the $800 \mathrm{~K}$ result. The two next nearest neighbor peaks near $5 \AA$ are starting to come together at $1050 \mathrm{~K}$, while the longer distance peaks are smoothing out and dropping away. The velocity auto correlation function for Ag at $1050 \mathrm{~K}$ is shown in the SI. We see that the correlations dissipate within $2.5 \mathrm{ps}$. The bond orientation order parameter for silver is shown in the SI. Strong orientation order is observed at $1050 \mathrm{~K}$.

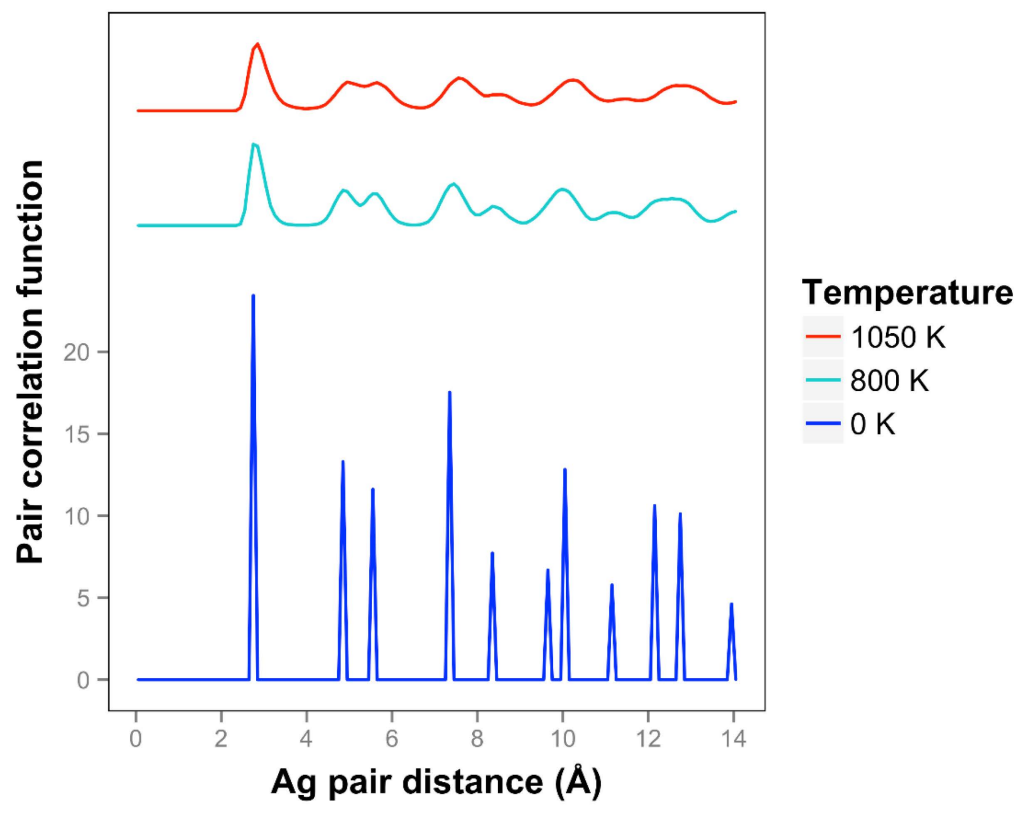

Figure 9. Pair correlation function for silver quasi-2D monolayers at temperatures ranging from $0 \mathrm{~K}$ to 1050 K. Higher temperatures are offset for visibility.

\subsection{Copper}

Snapshots from the $1320 \mathrm{~K}$ and $1400 \mathrm{~K}$ simulations are shown in Figure 10. Slow diffusion is observed, with an RMSD of $2 \AA$ and $1.8 \AA$ over times of 7.2 ps and 5.5 ps for 1320 and $1400 \mathrm{~K}$ respectively. This leads to a prediction of the diffusion coefficient of $\mathrm{D}=0.14$ for $1320 \mathrm{~K}$ and $\mathrm{D}=0.15$ for $1400 \mathrm{~K}$. This diffusion for the freestanding quasi-2D copper monolayer demonstrates clear quasi-2D liquid behavior. This quasi-2D liquid behavior can be observed in a movie of the $1400 \mathrm{~K}$ simulation in the SI. 


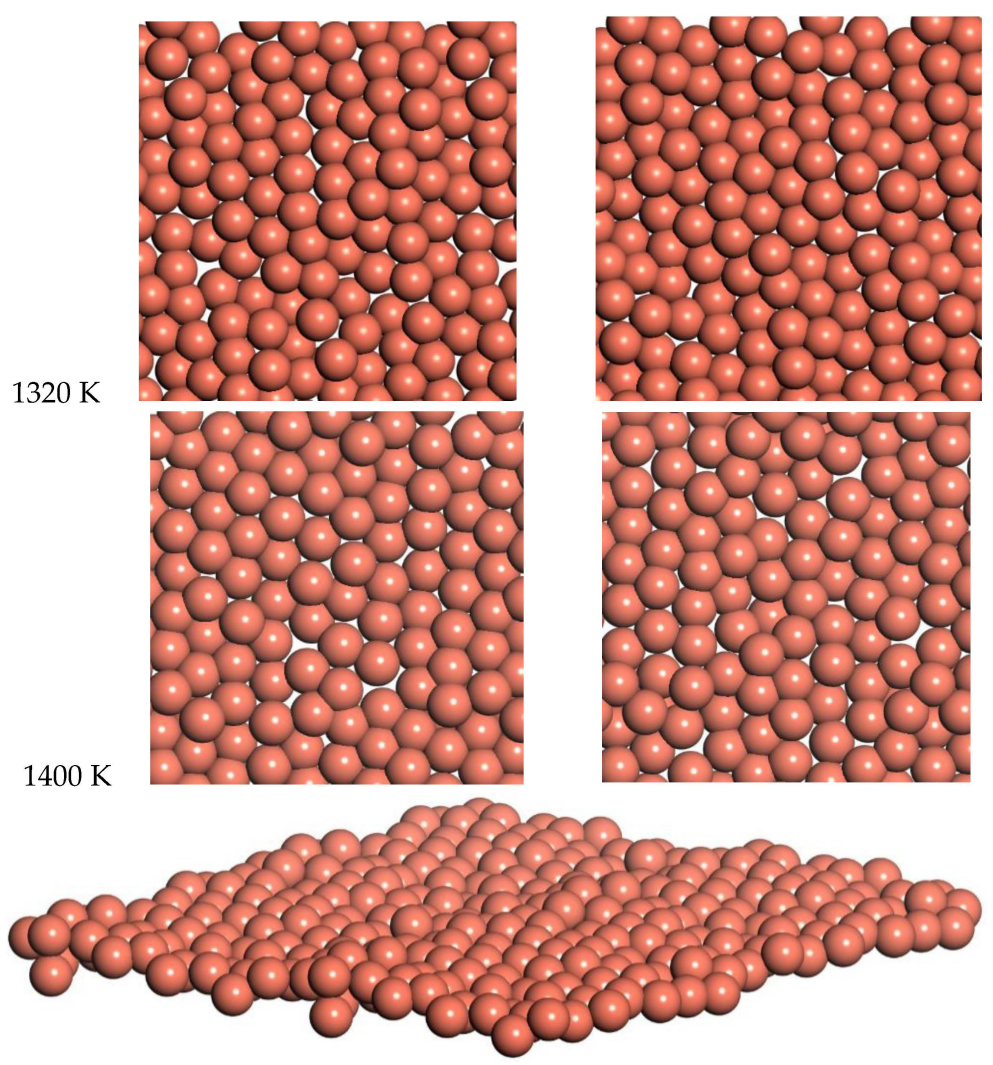

Figure 10. Molecular dynamics snapshot of quasi-2D Cu layer. Two snapshots separated by 0.2 ps are shown at $1320 \mathrm{~K}$ and $1400 \mathrm{~K}$. Angle view shown at $1400 \mathrm{~K}$.

The pair correlation function for copper is shown in Figure 11 and is consistent with quasi-2D melting at the higher temperatures of 1320 and $1400 \mathrm{~K}$. We see that two next nearest neighbor peaks between 4 and $5 \AA$ merge together at $1320 \mathrm{~K}$. The average number of bonds for $\mathrm{Cu}$ at $1400 \mathrm{~K}$ is 6.1 , slightly higher than the $0 \mathrm{~K}$ value due to the presence of some $3 \mathrm{D}$ bonds.

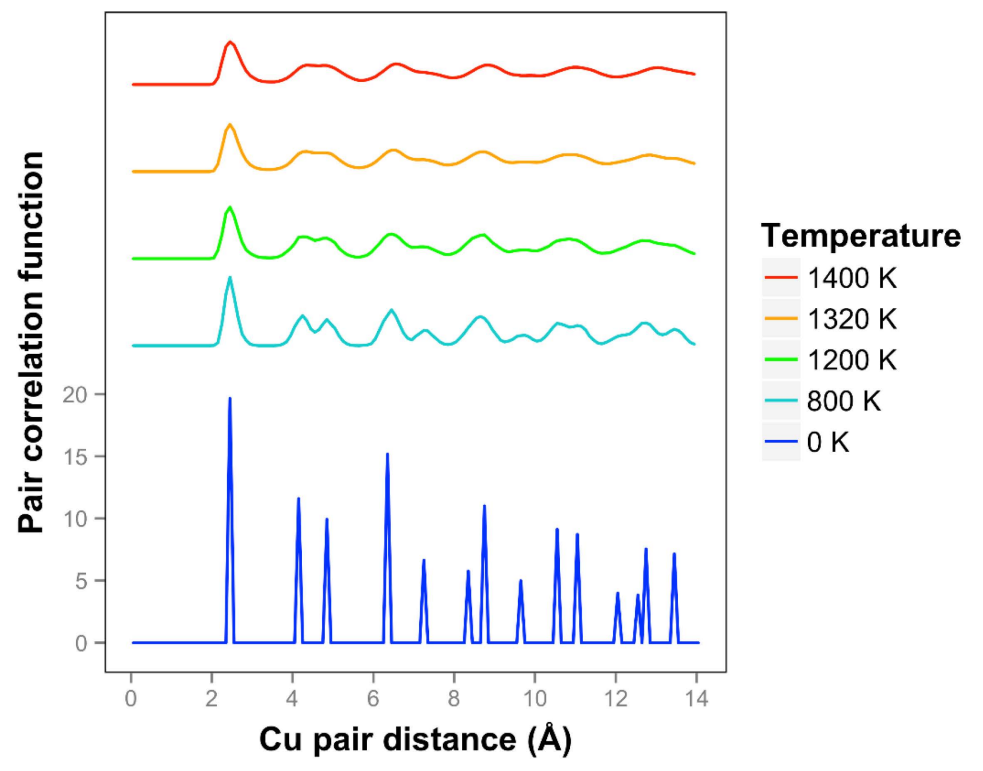

Figure 11. Pair correlation function for freestanding copper quasi-2D monolayers at temperatures ranging from $0 \mathrm{~K}$ to $1400 \mathrm{~K}$. Higher temperatures are offset for visibility. 
One can compare the temperature ranges for the freestanding quasi-2D monolayers to the bulk melting points for these elements. We compare these results in Table S3. We find that the temperature ranges are relatively close to the bulk melting points with variations of $-3 \%,-15 \%, 5 \%$, and $13 \%$, respectively, for $\mathrm{Cu}, \mathrm{Ag}, \mathrm{Au}$, and Pt. In particular, the Pt monolayer is stable up to very high temperatures.

We have used the highly reliable DFT-D ab initio method to study these systems. We feel that it is important to set a baseline using a reliable theory so that future work to extend the size or length of the simulations has a sound starting point. This work could be extended to other materials, or alloys. Our careful baseline study is not long enough or large enough to answer the question whether hexatic phase or multiple liquid phases exist in these systems, and so we will leave these questions for future studies.

\section{Conclusions}

In summary, we have seen that platinum, silver, gold, and copper can form stable freestanding quasi-2D liquid layers in freestanding monolayers at 2300-2400 K, $1050 \mathrm{~K}, 1600 \mathrm{~K}$, and 1320-1400 K, respectively. Planarizing forces stabilize the quasi-2D layer. The use of high quality DFT-D theory provides a substantial temperature correction of $20 \%$ compared to the previous calculations. The use of these dispersion corrections in the DFT calculation is crucial for these systems due to the very long bond length extensions observed. Remarkably, we observe a $4 \%$ reduction in the Pt maximum bond length location as the system enters the liquid state at $2400 \mathrm{~K}$. We also see a substantial change in the spring constant from 4.2 to $2.8 \mathrm{eV} / \AA^{2}$ for Pt as it goes into the liquid state. Pair correlation functions and diffusion measurements reveal liquid behavior in specific temperature ranges. The pair correlation function drops in the liquid state, while orientation order is reduced to a lesser degree.

Supplementary Materials: The following are available online at www.mdpi.com/2410-3896/1/1/1/s1 Molecular dynamics movie files of the platinum, gold, silver and copper quasi-2D liquid state are available. Side views, some ball and stick models of the molecular dynamics simulations, more computational details, RMSD plots, and analysis tables are also available. In particular, see Figure S1. Platinum $2400 \mathrm{~K}$ side view, and ball and stick model; Figure S2. Side views of Au models at 500, 800, 1200, 1400, and 1600 K; Figure S3. Last frame of gold simulation at $2000 \mathrm{~K}$, top view and ball and stick model of gold at $1600 \mathrm{~K}$; Figure S4. Silver $1050 \mathrm{~K}$ angle view; Figure S5. Copper side view at 1400 K; Figure S6. Plot of copper root mean square displacement versus time; Figure S7. Plot of Au root mean square displacement versus time; Figure S8. Plot of Ag root mean square displacement versus time; Figure S9. Plot of Ag velocity auto correlation function at $1050 \mathrm{~K}$; Figure S10. Plot of Pt velocity auto correlation function at $2400 \mathrm{~K}$; Figure S11. Orientation correlation function g6(r) for Au at various temperatures; Figure S12. Orientation correlation function $\mathrm{g} 6(\mathrm{r})$ for $\mathrm{Ag}$ at various temperatures; Figure S13. Bond length distribution for silver at $800 \mathrm{~K}$ and $1050 \mathrm{~K}$; Table S1. Simulation times and skip times for MD runs; Table S2. RMSD and Diffusion Constant D Values after Equilibration; and Table S3. Comparison of 2D Liquid Range to Bulk Melting Points for all materials. Supplementary videos are available at http:/ /dx.doi.org/10.5281/zenodo.46838 Video S1. 5 ps Molecular Dynamics Movie of Pt Freestanding Monolayer at 2400 K. Video S2. 6 ps Molecular Dynamics Movie of Ag Freestanding Monolayer at 1050 K. Video S3. 4 ps Molecular Dynamics Movie of Au Freestanding Monolayer at 1600 K. Video S4. 3 ps Molecular Dynamics Movie of Cu Freestanding Monolayer at $1400 \mathrm{~K}$.

Acknowledgments: We thank the Minnesota Supercomputing Institute (MSI) at the University of Minnesota for providing supercomputing resources. The molecular dynamics simulations were carried out on the Mesabi supercomputer at the MSI. L.M.Y. acknowledges support from startup funding from Huazhong University of Science and Technology. Research reported in this publication was supported by the National Institutes of Health under award T32-DK007158 (A.G.). The content is solely the responsibility of the authors and does not necessarily represent the official views of the National Institute of Diabetes and Digestive and Kidney Diseases (NIDDK) or the National Institutes of Health.

\section{References}

1. Zhao, J.; Deng, Q.; Bachmatiuk, A.; Sandeep, G.; Popov, A.; Eckert, J.; Rümmeli, M.H. Free-standing single-atom-thick iron membranes suspended in graphene pores. Science 2014, 343, 1228-1232.

2. Koskinen, P.; Korhonen, T. Plenty of motion at the bottom: Atomically thin liquid gold membrane. Nanoscale 2015, 7. [CrossRef] [PubMed]

3. Special issue on 2D Nanomaterials beyond Graphene. Acc. Chem. Res. 2015, 48, 119-127. 
4. Zheng, X.H.; Earnshaw, J.C. On the Lindemann criterion in 2D. Europhys. Lett. 1998, 41, 635-640. [CrossRef]

5. Zahn, K.; Maret, G. Dynamic Criteria for Melting in Two Dimensions. Phys. Rev. Lett. 2000, 85, 3656-3659. [CrossRef] [PubMed]

6. Zakharchenko, K.V.; Fasolino, A.; Los, J.H.; Katsnelson, M.I. Melting of graphene: From two to one dimension. J. Phys.: Condens. Matter 2011, 23. [CrossRef] [PubMed]

7. Singh, S.K.; Neek-Amal, M.; Costamagna, S.; Peeters, F.M. Rippling, buckling, and melting of single-and multilayer $\mathrm{MoS}_{2}$. Phys. Rev. B 2015, 91. [CrossRef]

8. Los, J.H.; Zakharchenko, K.V.; Katsnelson, M.I.; Fasolino, A. Melting temperature of graphene. Phys. Rev. B 2015, 91. [CrossRef]

9. Martínez-Guajardo, G.; Cabellos, J.L.; Díaz-Celaya, A.; Pan, S.; Islas, R.; Chattaraj, P.K.; Heine, T.; Merino, G. Dynamical behavior of Borospherene: A Nanobubble. Sci. Rep. 2015, 5. [CrossRef] [PubMed]

10. Kosterlitz, J.M.; Thouless, D.J. Ordering, metastability and phase transitions in two-dimensional systems. J. Phys. C Solid State Phys. 1973, 6, 1181-1203. [CrossRef]

11. Young, A.P. Melting and the vector Coulomb gas in two dimensions. Phys. Rev. B 1979, 19. [CrossRef]

12. Nelson, D.R.; Halperin, B.I. Dislocation-mediated melting in two dimensions. Phys. Rev. B $1979,19$. [CrossRef]

13. Bernard, E.P.; Krauth, W. Two-step melting in two dimensions: First-order liquid-hexatic transition. Phys. Rev. Lett. 2011, 107. [CrossRef] [PubMed]

14. Qi, W.; Gantapara, A.P.; Dijkstra, M. Two-stage melting induced by dislocations and grain boundaries in monolayers of hard spheres. Soft Matter 2014, 10, 5449-5457. [CrossRef] [PubMed]

15. Yang, L.-M.; Frauenheim, T.; Ganz, E. The new dimension of silver. Phys. Chem. Chem. Phys. 2015, 17, 19695-19699. [CrossRef] [PubMed]

16. Yang, L.-M.; Dornfeld, M.; Frauenheim, T.; Ganz, E.D. Glitter in 2D monolayer. Phys. Chem. Chem. Phys. 2015, 17, 26036-26042. [CrossRef] [PubMed]

17. Kittel, C. p.50 Introduction to Solid State Physics, 8th ed.; John Wiley \& Sons, Inc: Hoboken, NJ, USA, 2005.

18. Lide, D.R. CRC Handbook of Chemistry and Physics; CRC Press: Boca Raton, FL, USA, 2008; Volume 88.

19. Clark, S.J.; Segall, M.D.; Pickard, C.J.; Hasnip, P.J.; Probert, M.J.; Refson, K.; Payne, M.C. First principles methods using CASTEP. Z. Krist. 2005, 220, 567-570. [CrossRef]

20. Martyna, G.J.; Klein, M.L.; Tuckerman, M. Nosé-Hoover chains: The canonical ensemble via continuous dynamics. J. Chem. Phys. 1992, 97. [CrossRef]

21. R Core Team. R: A Language and Environment for Statistical Computing; R Foundation for Statistical Computing: Vienna, Austria; Available online: http://www.R-project.org.

22. Python Software Foundation. Python Language Reference, version 2.7. Available online: http:/ / www.python.org.

23. Oshima, Y.; Onga, A.; Takayanagi, K. Helical gold nanotube synthesized at 150 K. Phys. Rev. Lett. 2003, 91, 205503. [CrossRef] [PubMed]

(C) 2016 by the authors; licensee MDPI, Basel, Switzerland. This article is an open access article distributed under the terms and conditions of the Creative Commons by Attribution (CC-BY) license (http://creativecommons.org/licenses/by/4.0/). 